\title{
Pêche, conservation environnementale et aire protégée
}

Marginalisation socio-économique et négociations de l'espace littoral au sein d'une réserve de biosphère au Yucatán, Mexique

Negotiating fisheries, environmental conservation and protected areas. Spatial and socio-economic marginalization along the coastal area of the Ría Lagartos Biosphere Reserve in Yucatán, Mexico

\section{Sabrina Doyon et Pierre-Alexandre Paquet}

\section{OpenEdition}

\section{Journals}

Édition électronique

URL : http://journals.openedition.org/ethnoecologie/3641

DOI : $10.4000 /$ ethnoecologie.3641

ISSN : 2267-2419

Éditeur

Laboratoire Eco-anthropologie et Ethnobiologie

Référence électronique

Sabrina Doyon et Pierre-Alexandre Paquet, " Pêche, conservation environnementale et aire protégée », Revue d'ethnoécologie [En ligne], 14 | 2018, mis en ligne le 31 décembre 2018, consulté le 20 avril 2019. URL : http://journals.openedition.org/ethnoecologie/3641 ; DOI : 10.4000/ethnoecologie.3641

Ce document a été généré automatiquement le 20 avril 2019.

\section{(c)}

Revue d'ethnoécologie est mis à disposition selon les termes de la licence Creative Commons Attribution - Pas d'Utilisation Commerciale - Pas de Modification 4.0 International. 


\section{Pêche, conservation environnementale et aire protégée}

Marginalisation socio-économique et négociations de l'espace littoral au sein d'une réserve de biosphère au Yucatán, Mexique

Negotiating fisheries, environmental conservation and protected areas. Spatial and socio-economic marginalization along the coastal area of the Ría Lagartos Biosphere Reserve in Yucatán, Mexico

Sabrina Doyon et Pierre-Alexandre Paquet

\section{Introduction : Las Coloradas, une oasis dans les salines?}

Sis sur une mince bande de terre s'allongeant finement entre la mer des Caraïbes et la lagune de Ría Lagartos, le village de Las Coloradas est comme une petite oasis au milieu des bassins d'évaporation de sel et de la plage de sable blanc. Ces bassins d'évaporation qui se déclinent en camaïeux de rose et d'orangé ont d'ailleurs donné son nom au village. Situé sur la côte nord-est de l'état du Yucatán, Las Coloradas est un village typique parmi les quatre de la réserve de biosphère de Ría Lagartos. Il compte 1151 habitants répartis dans 280 unités résidentielles (INEGI 2010). Son contexte est marqué par la saisonnalité de la pêche côtière en décroissance, l'insertion dans le réseau des réserves de biosphère et des activités de conservation, l'offre écotouristique naissante avec l'observation des flamants roses et les migrations pendulaires des travailleurs vers les grands centres urbains. Le village se distingue cependant des autres villages de la côte par un élément très important : la production industrielle de sel. Son histoire est en effet étroitement liée aux caractéristiques géophysiques de la côte yucatèque qui permet la production de sel par évaporation (Lapointe 2006). 
Figure 1 : Carte de situation

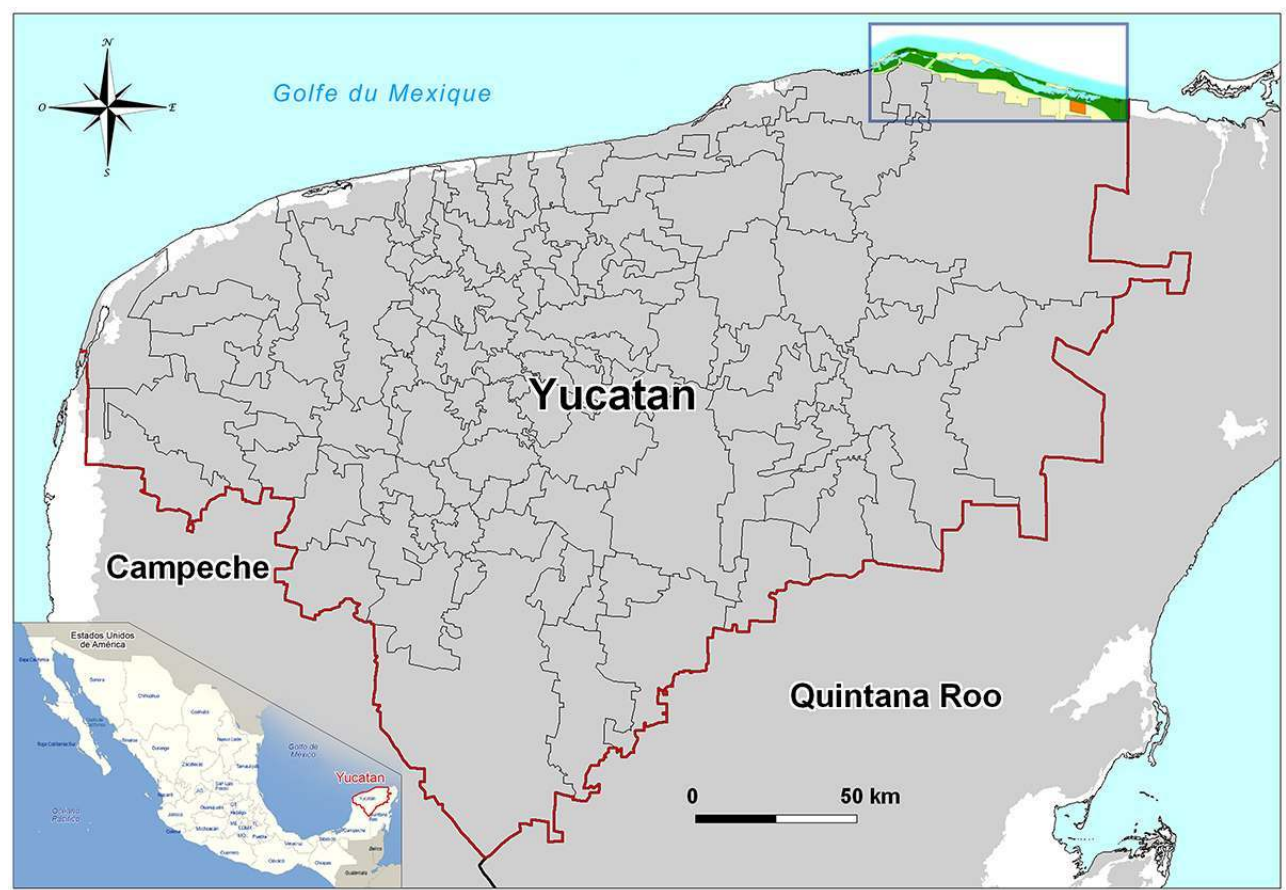

Figure 2 : Carte officielle de la zone

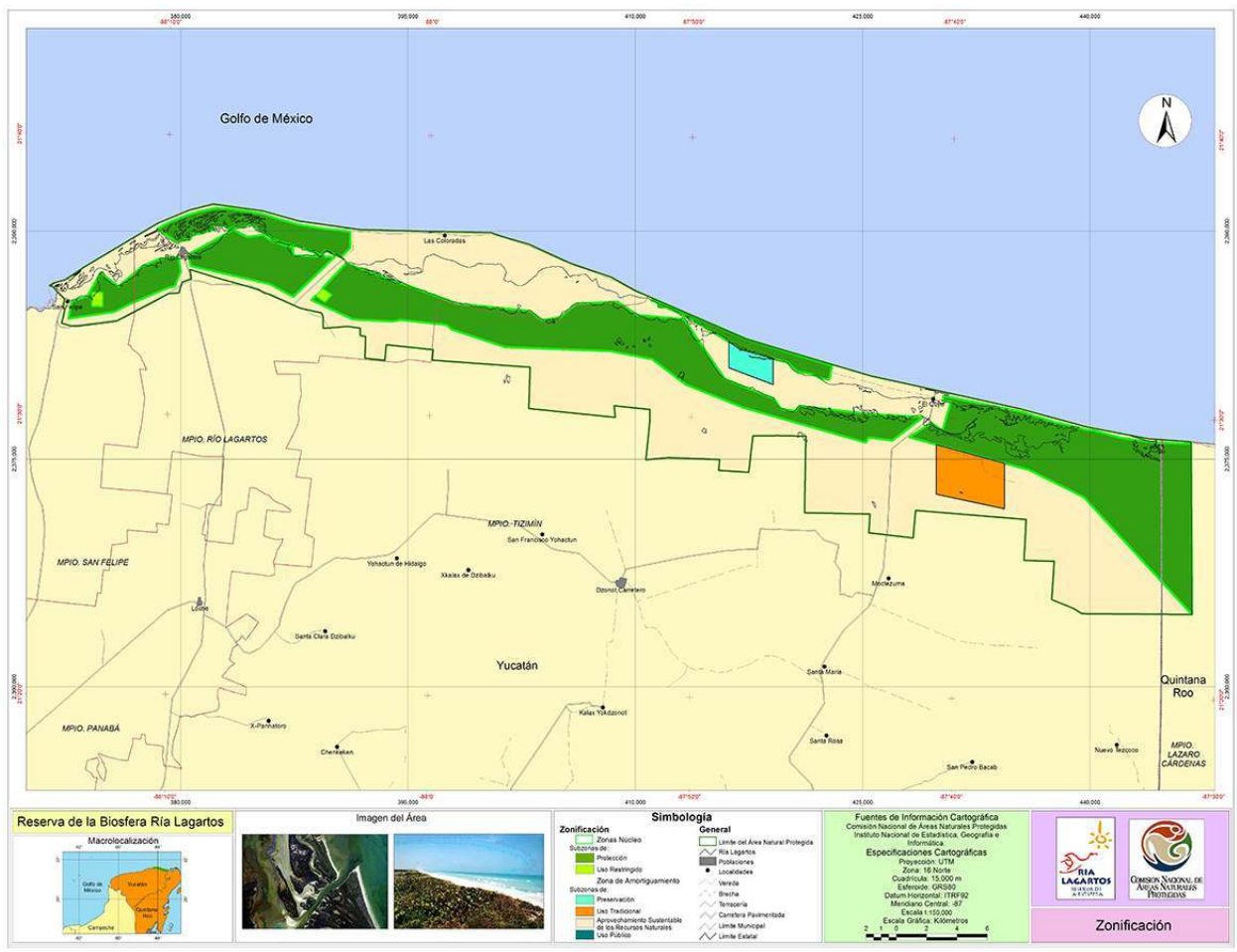

2 Las Coloradas offre une perspective unique et révélatrice des mécanismes à l'œuvre dans la construction de l'espace côtier, illustrant comment des changements d'usage et de contrôle peuvent s'y dérouler par le truchement des revendications d'accès, des appropriations et des négociations de l'espace entre les différents acteurs en place 
(pêcheurs, paludiers, représentants de la conservation, entrepreneurs touristiques). Ces dynamiques articulent les intérêts de divers groupes d'acteurs et s'ancrent dans des rapports de pouvoir définissant les trois pôles de cet espace: des lieux définis par les activités de pêche, ceux des paludiers dans la saline industrielle et la place donnée à la conservation et la mise en valeur de l'environnement (Réserve de biosphère).

La political ecology s'intéresse à la façon dont les rapports de pouvoir façonnent les relations entre les humains et la nature (Wolf 1972, cité dans Biersack \& Greenberg 2006). Ces liens peuvent être examinés avec plus de précision par trois angles analytiques: l'impact socio-environnemental du développement capitaliste, les implications sociales et politiques de la conservation environnementale et l'économie politique de la production de nouvelles natures, de nouveaux paysages et espaces (Peet et al. 2011). Ces angles soulèvent à leur tour des questions de gouvernance environnementale, de représentations de la nature, d'historicité et d'échelles.

4 Les rapports socio-environnementaux des habitants de Las Coloradas sont marqués par le contexte historique et des structures plus larges. Les négociations pour l'accès, l'usage et le contrôle de la nature et de l'espace côtier s'arriment aux activités productrices ayant cours au village, dont le développement parfois idiosyncratique demeure néanmoins sous l'influence de l'économie politique mexicaine conjuguant politiques étatiques de développement ainsi que production capitaliste mondialisée. Les sections suivantes examinent comment l'espace côtier a été marqué par les enjeux propres à la production du sel, à la pêche et à la gestion et la conservation environnementales dans un contexte et une histoire dynamiques.

Les données présentées ici reposent sur des recherches de terrain qui se sont déroulées à las Coloradas durant les mois de juin et juillet de 2006, 2007 et 2008 dans le cadre d'un projet de recherche plus vaste examinant la construction de l'espace côtier dans les six villages des deux réserves de biosphère de la côte du Yucatán, Ría Lagartos et Celestun, entre 2003 et 2011. Plusieurs méthodes d'enquêtes ont été employées spécifiquement à Las Coloradas : des observations participantes, la consultation des archives locales et des documents des représentants politiques municipaux, des entrevues informelles et 31 entrevues semi-dirigées enregistrées avec des pêcheurs, des travailleurs de l'usine de sel, des participants à des projets environnementaux locaux et des employés de la Réserve.

\section{Las Coloradas : avènement d'un village parmi les salines}

Des évidences archéologiques prouvent que le sel de Las Coloradas était déjà d'une grande importance à l'époque précolombienne maya (Lapointe 2006). À l'époque coloniale toutefois, l'exploitation du sel dans la région n'a eu lieu que de manière intermittente et y constituait une des activités de subsistance parmi d'autres, principalement la coupe de bois. Il faudra attendre les années 1930 avant de voir, sous la direction de la famille yucatèque Roche, une reprise complète de l'industrie du sel, avec un début modeste, mais une modernisation et une croissance rapides.

7 L'entreprise des Roche à Coloradas, enregistrée en 1946 sous le nom d'Industria Salinera de Yucatán (ISYSA), est rapidement devenue la plus importante raffinerie de sel de l'État du Yucatán et la deuxième en importance au Mexique. Au début de l'exploitation, la famille Roche a fondé un rancho ${ }^{1}$ sur ses terres de Las Coloradas. Elle y a logé ses ouvriers, 
une main-d'œuvre masculine qui n'était pas recrutée dans l'économie dominante du henequen (variété de sisal). Nos recherches généalogiques dans le village révèlent que la majorité des familles viennent de l'ouest de l'État, particulièrement de Dzindzantun, Ixil, et Izamal. L'immigration dans le village pour travailler le sel s'est poursuivie jusque dans les années 1970. La langue maya est toujours parlée couramment dans plusieurs des familles du village.

Exploitant 2800 hectares au cœur d'une concession en totalisant 5000 , l'entreprise des Roche emploie aujourd'hui $80 \%$ des hommes de Las Coloradas. L'industrie salinière a été intégrée dans la Réserve de biosphère de Ría Lagartos et se trouve donc entourée des zones dédiées à la conservation de la biodiversité. Cette configuration particulière de Las Coloradas, impliquant une concession salinière inscrite à l'intérieur d'une Réserve de biosphère, est un cas unique dans la péninsule du Yucatán.

9 Afin de comprendre comment l'espace est investi à Las Coloradas, la question du sel est de premier ordre. Les premiers arrivants dans les années 1940 s'installèrent dans les dortoirs (galeras) construits par les Roche. Rejoints plus tard par leur famille, ces travailleurs firent construire des maisons individuelles sur les terrains de la compagnie. Leur syndicat, fondé en 1977, a d'ailleurs contribué à ce que chacune des familles des travailleurs obtienne une maison. Les lots sur lesquels ces maisons se trouvent sont toutefois demeurés propriété de la compagnie. Jusqu'à récemment, la compagnie fournissait tous les services, dont l'eau et l'électricité. Elle assurait également l'offre de biens de consommation, étant propriétaire de l'épicerie et de la cantine du village où les gens pouvaient payer en coupons qu'elle leur émettait, ou acheter à crédit, la somme due étant plus tard prélevée directement sur le salaire. ISYSA assurait également le service d'éducation primaire aux enfants. Enfin, le fonctionnement de la vie dans le village était étroitement surveillé. Les habitants disent qu'ils devaient se soumettre aux règles de l'entreprise autant au travail que dans la communauté. Comme en témoigne un employé de l'usine :

« il y avait moins de liberté parce que, par exemple, s'ils (les gardiens de sécurité de

l'usine) te voyaient te promener à minuit dans le village, les soldats te demandaient

ce que tu faisais-là... c'est clair, il y avait plus de surveillance. »

10 Il y a plus de 60 ans, la production se faisait à puros brazos, comme le racontent les habitants, c'est-à-dire entièrement grâce l'énergie humaine. Les bassins saliniers, connus de manière générique sous le nom de charcas, bien qu'ils aient chacun leur nom propre, étaient travaillés à la pelle et le sel ensaché manuellement. Entre 1946 et 2015, la production a centuplé et les hectares travaillés ont été multipliés par près de 30. Le seul hiatus dans la production fut causé par le passage dévastateur de l'ouragan Gilberto en 1988. L'usine de sel a alors subi des dommages majeurs et une centaine d'employés furent mis à pied. Ceux-ci purent toucher des primes et nombre d'entre eux constituèrent alors des associations de pêcheurs ${ }^{2}$. Aujourd'hui, l'usine fonctionne nuit et jour, les ouvriers travaillent des quarts de huit heures qui changent chaque semaine, afin de distribuer les quarts de nuit entre tous.

11 Le modernisme des équipements industriels de l'entreprise est source de fierté au village. Même ceux qui ne sont pas employés par ISYSA connaissent avec précision les taux de production annuels et peuvent les réciter savamment. Tous mettent d'ailleurs cette modernisation en parallèle avec les gains syndicaux qui ont bonifié les salaires, les conditions de travail, les bénéfices marginaux, les retraites et, dans une certaine mesure, l'accès à des maisons en dur pour les employés de l'usine. Le travail dans l'usine est très 
prisé, et les places disponibles sont attribuées en fonction d'une liste d'attente où prédominent les noms des fils des travailleurs, des hommes.

La production de l'usine - seize dérivés de sel - est principalement destinée au marché mexicain, et des dizaines de camions semi-remorques de 40 tonnes entrent chaque semaine au village pour charger le sel avant de l'emmener dans d'autres états de la République. Pendant leur courte escale, il arrive que les camionneurs achètent des produits locaux, poissons, hamacs ou œufs de tortues marines ${ }^{3}$.

13 Une majorité de la population se sent privilégiée de vivre à Las Coloradas et les habitants considèrent que la qualité de vie et de l'environnement y est exceptionnelle et incommensurable avec la réalité des autres villages et des grands centres. Les employés de l'usine de sel apprécient de pouvoir bénéficier de cette source d'emplois stables et rémunérateurs. Les habitants qui n'y travaillent pas, dont les pêcheurs se sentent cependant laissés pour compte, voire coincés au bout de la route sans issue menant au village: Las Coloradas n'est pas pour eux une oasis. Ils aspirent à s'approprier le territoire, du moins en partie, afin d'y développer d'autres activités rémunératrices, comme l'écotourisme, des alternatives à l'économie dominante basée sur le sel et la pêche en déclin.

14 Cette aspiration à investir et s'approprier l'espace s'ancre à Las Coloradas dans la complexe, et parfois nébuleuse, question foncière. Le village fait maintenant partie du municipe de Río Lagartos, qui assure certains services municipaux, de voirie et de sécurité civile. Toutefois, la question de la propriété foncière, incluant les responsabilités et des bénéfices qui en découlent, demeure complexe. En effet, la compagnie salinière demeure propriétaire de la majeure partie des terres à l'exception de rares parcelles individuelles et malgré la municipalisation du village, c'est l'entreprise qui continue de faire l'entretien des rues et des infrastructures du village et elle refuse de concéder ou de vendre des parcelles de terrain aux habitants. La situation de la propriété n'est pas claire aux yeux de plusieurs, et nous avons souvent entendu que les « Roche sont propriétaires de tout $»^{4}$.

15 Les possibilités d'utilisation du territoire sont aussi contraintes par la réserve de biosphère qui limite les types d'actions, d'usage et d'appropriation possibles. Toutes les personnes que nous avons rencontrées disent souhaiter que le village devienne autonome, libre, un municipio libre, dégagé du contrôle territorial de l'usine, de la réserve et de la municipalité de Río Lagartos qui, opinent-elles, gèrent le village dans leur seul intérêt. 
Figure 3 : Colline de sel, résultat de la production de l'entreprise salinière ISYSA, protégée des eaux de la mer et de la lagune par une digue de sable

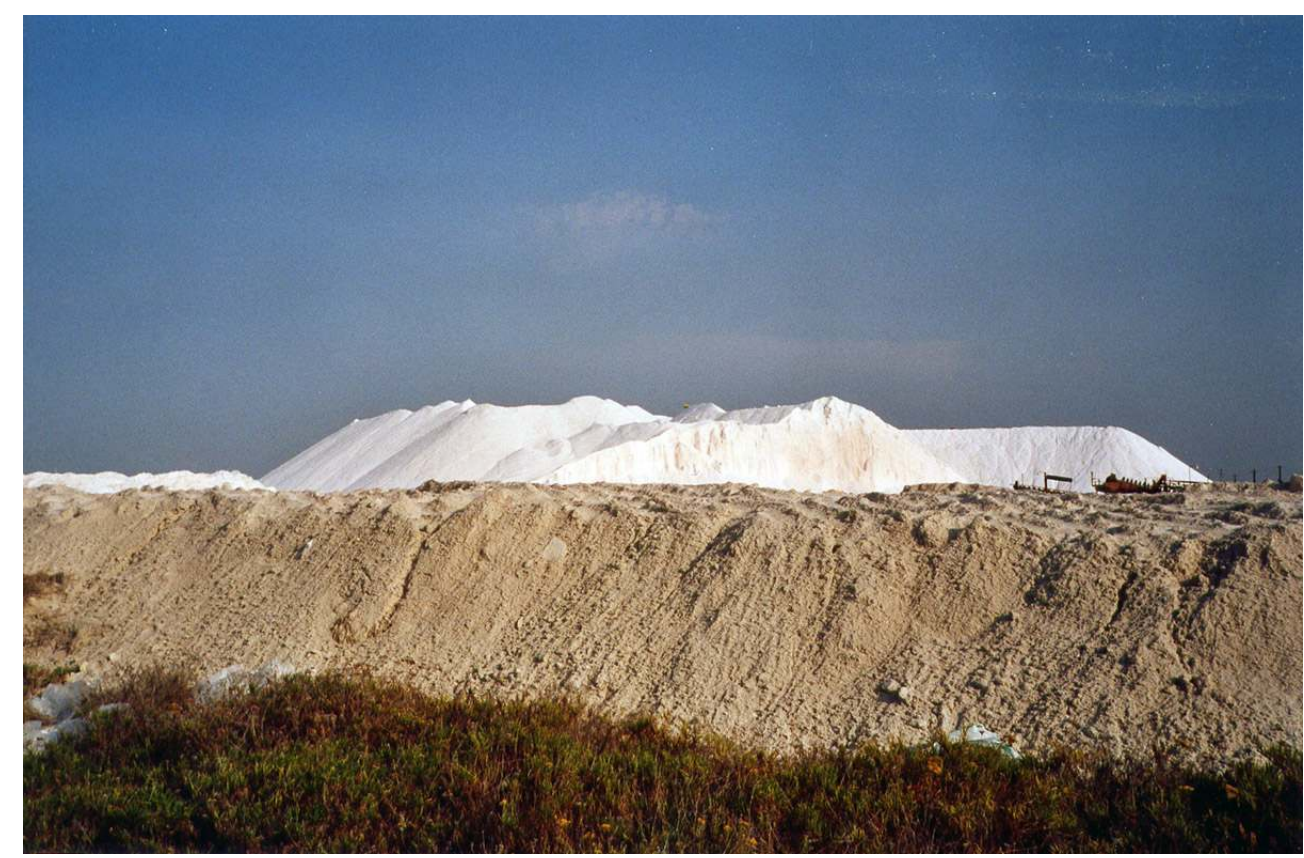

(c) S. Doyon

\section{La pêche à Las Coloradas : entre salines et politiques}

Au Yucatán, la pêche côtière est demeurée relativement marginale jusque dans les années 1960. La production commerciale annuelle était alors d'environ 1000 tonnes. C'est au tournant des années 1970 qu'elle prit son essor, notamment à l'incitation du gouvernement dans un contexte où l'industrie du hénequen s'effondrait. Elle s'est ensuite rapidement affirmée comme l'une des activités économiques principales de l'État (Doyon et al. 2008, Fraga 1999 et 2004, Fraga et al. 2008).

S'inspirant du cadre de développement économique hérité du sexennat de Cárdenas trente ans plus tôt (1934-1940), les gouvernements ont mis sur pied, au Yucatán comme ailleurs, des coopératives de pêche dotées de privilèges exclusifs tels des concessions (des droits exclusifs sur des espèces à haute valeur commerciale telles la langouste, la crevette et certains mollusques à coquille), du financement pour la construction d'infrastructures et du crédit pour l'achat d'équipements de pêche.

L'« âge d'or » des pêcheries yucatèques a duré jusqu'aux années 1990 avec des captures de 20000 tonnes par an et l'emploi de près de 20000 pêcheurs. Toutefois, le virage néolibéral des politiques gouvernementales depuis 1980 a eu pour effet de, progressivement, marginaliser socialement, économiquement et politiquement la pêche côtière du Yucatán et son secteur coopératif (Fraga 1999 et 2004, Fraga et al. 2008). Au cours de ces réformes, l'ancien ministère des Pêches (SEPESCA) a été dissout et la tenure maritime a été transformée en éliminant, notamment, les droits exclusifs de pêche que détenaient les coopératives. Depuis, ces dernières sont en compétition avec les secteurs privé et industriel pour les permis, l'accès et la commercialisation de ces espèces. En plus de causer des pertes économiques aux coopératives, ces changements apportés aux droits 
d'accès exacerbent des enjeux relevant de l'administration interne des coopératives, précipitant leur démantèlement (Fraga et al. 2008).

Depuis 1990, le secteur des pêches est aussi affecté par la dégradation environnementale et la diminution des prises, (Fraga 1999, Fraga et al. 2008) un phénomène qui n'est pas exclusif à la côte mexicaine (Mansfield 2001). Aujourd'hui, les pêcheurs côtiers du Yucatán témoignent que la pêche ne leur rapporte plus un revenu suffisant et satisfaisant. Dans ce contexte où la vulnérabilité des pêcheurs est croissante, où les politiques étatiques n'appuient plus le développement de la pêche côtière, où les pêches industrielles sont en croissance, où les écosystèmes se détériorent, les conflits et les luttes concernant l'accès, l'utilisation et le contrôle des ressources halieutiques semblent s'intensifier. Les conflits entre les regroupements de pêcheurs d'une même région - ou entre les pêcheurs locaux et ceux provenant d'autres régions - à propos des droits d'accès et de la légitimité de l'accès à une zone de pêche sont de plus en plus fréquents, une situation vécue à Coloradas.

Les pêcheurs que nous y avons rencontrés à Las Coloradas témoignent de ces transformations de l'espace côtier. À Las Coloradas, 30 \% des hommes pratiquent la pêche comparativement à $60 \%$ pour les autres villages côtiers de la région selon nos données. Bien que moins pratiquée, la pêche y demeure capitale pour les habitants du village. En raison de l'histoire du peuplement du village intimement liée à la production du sel, l'intérêt pour la pêche professionnelle s'y est développé plus tardivement qu'ailleurs dans la région. Par conséquent, le village ne dispose pas des infrastructures que l'État avait subventionnées ailleurs sur la côte dans les années 1970, tel un port ou une zone abritée pour les embarcations, qui sont donc disposées sur la plage. Les pêcheurs déplorent aussi que, contrairement aux employés de l'usine de sel, ils n'ont pas accès à une assurancetravail ou une pension. Cette comparaison aux paludiers (qui sont protégés et pensionnés par ISYSA) plutôt qu'aux pêcheurs des autres villages (qui n'ont pas plus accès à ce type d'avantages) est, selon nous, illustrative de l'importance des relations communautaires dans ce village. 
Figure 4 : Embarcations des pêcheurs de Las Coloradas hissées sur la plage ; convoyeur maritime de l'usine de sel en arrière-plan

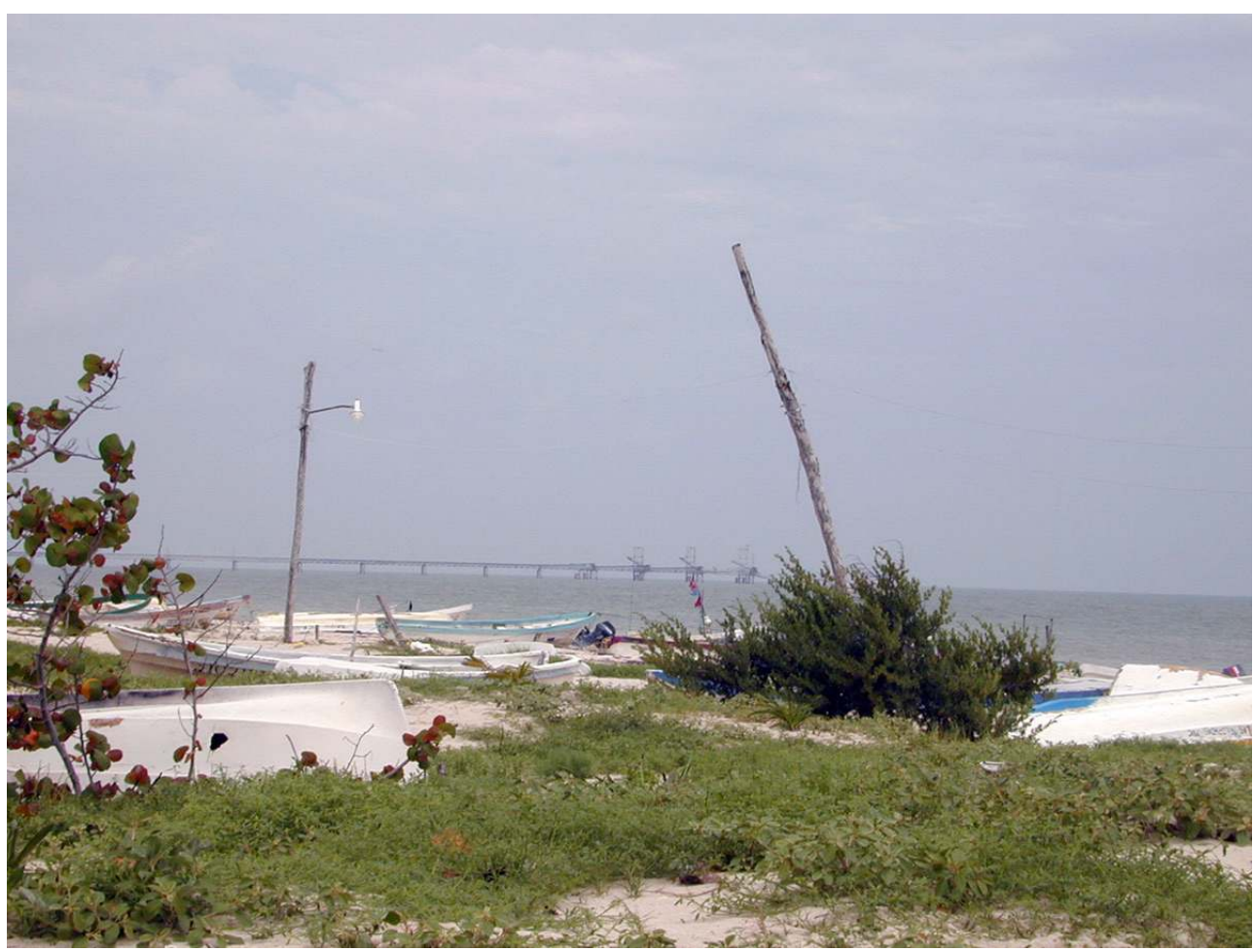

(C) S. Doyon

La pêche emploie plus de cent habitants de Las Coloradas sur une base permanente, et encore plus de façon saisonnière. Les espèces commerciales les plus importantes de la région de Las Coloradas sont les poissons à écailles, le poulpe et la langouste. L'une des pêches les plus pratiquées à Las Coloradas se fait à la palangre. La principale espèce visée par cette technique est le mérou (mero). Les pêcheurs âgés comparent les prises d'aujourd'hui à celles du temps où un court filin et un seul hameçon suffisaient. Selon eux, il est évident que la ressource décline tant par le nombre que par la taille des poissons capturés. La pêche au filet, elle aussi largement pratiquée, vise quant à elle le requin à nez pointu (cazón) et la sardine (sardina). Dans ce cas également, les pêcheurs constatent une diminution des ressources halieutiques. Parmi les causes possibles, les pêcheurs locaux évoquent les bateaux de pêche de plus grande capacité opérant au large, la compétition des pêcheurs des autres ports et les effets répétés et cumulatifs des tempêtes tropicales et des ouragans sur le milieu côtier.

Les pêcheurs de Las Coloradas doivent se déplacer à Río Lagartos afin d'y acheter la glace, le carburant pour leurs moteurs hors-bord et les appâts, ce qui implique des coûts importants qui s'ajoutent aux contraintes de la pêche, le nombre limité de permis, le déclin des stocks et la diminution des prix du poisson.

Le contexte de la pêche est difficile à Las Coloradas et certains sont prêts à se retirer. Déjà, ceux qui s'y dédiaient comme activité d'appoint se départissent de leur équipement. Les membres des trois coopératives ne s'en tirent pas nécessairement à meilleur compte, puisque l'État mexicain se désinvestit des pêcheries artisanales au profit de la pêche industrielle et de l'aquaculture. Certains avancent que la pêche n'est plus rentable et qu'il faudra trouver d'autres manières de gagner son pain, par exemple en investissant 
d'autres espaces dédiés à de nouvelles activités, comme l'affirme un pêcheur membre d'une coopérative :

«Les pêcheurs s'en vont, tout simplement. Il n'y a pas d'autre façon de faire. Beaucoup de pêcheurs sont partis, des gens qui étaient nés ici. Il y en a maintenant beaucoup qui s'en vont travailler à la maquiladora près de Tizimín. Un camion vient les chercher. Ils sont très peu payés et en plus, il y a le temps pour aller là-bas. Mon fils se lève à cinq heures le matin, il déjeune, je crois qu'il part à cinq heures et demie et il arrive là-bas vers six heures ou six heures et demie ».

La pêche à la langouste peut servir d'exemple illustrant la saturation de l'espace des pêcheries. Les coopératives de pêcheurs de Las Coloradas ne détiennent pas de permis de pêche à la langouste, ni les équipements appropriés à une pêche intensive, car c'est la Fédération régionale qui en détient l'exclusivité, or les pêcheurs professionnels de Las Coloradas ne sont pas membres de la Fédération régionale des sociétés coopératives de pêcheurs de l'État du Yucatán. En conséquence, les coopératives de Río Lagartos, San Felipe et El Cuyo peuvent pêcher la langouste légalement, notamment face au village de Las Coloradas, contrairement aux pêcheurs de Las Coloradas eux-mêmes. Par le passé, certains ont tenté d'adhérer aux coopératives de Río Lagartos afin de prendre part à la pêche à la langouste qui est très abondante dans leurs eaux. Aujourd'hui, ces pêcheurs témoignent en très mauvais termes de leur expérience. Non seulement les frais de transports entre Las Coloradas et Río Lagartos rendaient leur initiative peu rentable, mais les pêcheurs disent avoir été mal payés, voire exploités par leurs patrons.

Malgré cela, certains pêcheurs de Las Coloradas persistent à prélever de petites quantités de précieux crustacés lors d'incursions de pêche en apnée. Ils agissent ainsi en revendiquant des droits acquis sur cette pêche, mais risquent de fortes sanctions :

« Nous avons toujours travaillé de la pêche et je me suis battu durement pour ce qui en est de la langouste. Je considère que nous détenons le droit. Ce que nous souhaitons, ce n'est pas d'aller plonger à Río ou à San Felipe, mais seulement ici, entre nous. Ils nous ont mis six mois en prison, j'ai perdu mon embarcation, j'ai perdu mon moteur pour m'être battu au sujet du terrain où nous pourrions plonger $"$.

Les pêcheurs de Las Coloradas considèrent injuste et injustifiable l'interdiction de pêche qui leur est imposée. Ils analysent de plus que l'utilisation de compresseurs entraîne une surexploitation de la langouste alors que leur propre pratique de pêche en apnée est " écologique et biologique ".

Puisque les effectifs gouvernementaux manquent pour assurer un contrôle soutenu de la pêche aux langoustes, une surveillance est assumée par les pêcheurs eux-mêmes, particulièrement par les détenteurs de permis qui sont prompts à dénoncer les pêcheurs de Las Coloradas. Les pêcheurs se font aussi justice eux-mêmes. Le vol d'embarcations et le vandalisme figurent au nombre des représailles entre pêcheurs. La qualité des relations entre les villages pâtit donc suite au déclin de la ressource et des autres contraintes pesant sur la pêche.

Dans une moindre mesure, les problèmes rencontrés par les pêcheurs de Las Coloradas lors de la pêche à la langouste se répètent lors de la saison du poulpe. Et là encore, les pêcheurs se sentent démunis, sans appui institutionnel.

La pêche à gué, qui n'est pas prise en compte par les statistiques de la pêche professionnelle, représente également pour les autres travailleurs de Las Coloradas une façon de compléter leurs revenus et de mieux subvenir aux besoins de leur famille. Ces derniers pêchent principalement sur le pont qui enjambe l'embouchure de la lagune, 
quelques kilomètres à l'ouest du village. Ce lieu de pêche est toutefois disputé et revendiqué par des pêcheurs de Río Lagartos. Ces derniers disent que le pont «leur appartient" puisqu'il est situé dans la municipalité de Río Lagartos, alors que les Coloradeños affirment au contraire qu'il est à eux, puisque c'est le complexe industriel de fabrication de sel situé dans le village, qui l'a construit et qui en assure l'entretien. Il est intéressant ici de noter, une fois de plus, l'appartenance corporatiste revendiquée par les habitants de Las Coloradas les liant à l'entreprise salinière.

Notons que les expériences des pêcheurs de Las Coloradas à propos de la question des permis transforment les relations avec le municipe de Río Lagartos, qui est perçu comme en partie responsable de leur infortune par l'accaparement qu'il ferait des ressources et des permis.

\section{La Réserve de biosphère de Río Lagartos : entre projets à développer et protection à consolider}

Le Mexique est reconnu par la communauté internationale comme étant un pays doté d'une très grande biodiversité, laquelle représenterait près de $10 \%$ de la flore et de la faune mondiale et comprendrait entre $10 \%$ et $20 \%$ des espèces endémiques mondiales (Breunig 2006). Les aires protégées sont l'outil privilégié par l'État mexicain pour conserver ses écosystèmes naturels. L'état a, depuis les années 1990, institutionnalisé la conservation environnementale. La protection de la biodiversité s'inscrit dans sa structure bureaucratique depuis la création du ministère de l'environnement et des agences environnementales qui lui sont tributaires.

Sous l'impulsion de recommandations internationales et grâce au discours sur le développement durable, de nombreuses aires protégées ont été créées au Mexique. En 2014, il existait 176 aires protégées de juridiction fédérale au Mexique ; elles couvrent une superficie de 25394779 hectares équivalant à 12,92 \% du territoire national. La péninsule du Yucatán compte huit réserves de biosphère, huit parcs nationaux et six aires de protection de la faune et de la flore, couvrant $34827 \mathrm{~km}^{2}$ et équivalant à plus de $20 \% \mathrm{du}$ territoire de la Péninsule (CONANP 2010).

Les réserves de biosphère ont été choisies par le gouvernement comme moyen privilégié pour appliquer les mesures de conservation dans la région de Coloradas. Ces réserves se distinguent des autres modèles de conservation à deux niveaux. D'abord, l'habitation et les activités humaines y sont acceptées, contrairement à d'autres formes de protection tels les parcs nationaux mexicains qui les excluent. Ensuite, leur système de zonage permet d'articuler les objectifs de conservation à ceux du développement (UNESCO 1996).

Las Coloradas compte une station scientifique de la Réserve de biosphère située à l'extrémité est du village. $Y$ habite un biologiste dont les tâches sont d'assurer la surveillance de la nidification des tortues marines ainsi que le fonctionnement d'une pépinière consacrée à la production de plants de palétuviers pour la reforestation et diverses autres essences de palmiers vendus aux hôtels de la région et de la Riviera maya. Les activités de ce professionnel sont appuyées par des étudiants bénévoles ainsi que par des collègues de la station de Ría Lagartos, mais elles n'impliquent pas les habitants du village dans une même mesure, sauf en ce qui concerne la surveillance, la recension et la protection des tortues marines. 
Elle doit arpenter en quad chaque nuit la plage entre Las Coloradas et El cuyo, surveiller les tortues qui viennent y pondre et prélever les œufs afin de les mettre à l'abri des braconniers et des animaux prédateurs dans un enclos et des nids artificiels à la station de la Réserve. Cependant, cet emploi n'est pas stable et il est parfois suspendu. Il semble que ce soit le cas lorsque des étudiants en stage dans la région font le travail gratuitement. Un employé de la réserve explique :

«Nous avons décidé de leur enlever leur emploi parce qu'il y avait deux ou trois personnes qui faisaient un mauvais usage du matériel pour aller se balader avec leur famille, donc ils disposaient d'une ressource qui n'est même pas la nôtre, c'est celle du gouvernement. Nous avons donc décidé d'engager des gens professionnels avec une éthique pour surveiller le travail. En fait, nous avons des volontaires qui viennent en vacances, des jeunes hommes et des jeunes femmes de l'université et ils viennent gratuitement. Ils s'offrent de le faire gratuitement; nous leur fournissons la nourriture, l'hébergement et le transport. Parfois ils restent un mois, deux, trois mois. Nous n'avons pas à les payer. Et eux, ils font les choses comme il faut, sans autre intérêt. Les gens de Coloradas veulent être payés et en plus, ils entachaient la réputation de Las Coloradas ».

Cette vision et les valeurs qui la fondent sont certainement déterminantes dans les relations qu'entretient la Réserve avec les habitants de Las Coloradas, qui eux jugent que leur temps investi mérite d'être rémunéré, contrairement à ce que semble penser les employés de la Réserve.

Réserve administre d'autres programmes d'emplois temporaires (PET) et de développement régional durable (PRODERS) au sein des villages de la Réserve. Selon ce que nous en ont dit les responsables de la Réserve, ces programmes sont intimement liés aux saisons de pêche et proposent un revenu alternatif aux familles de pêcheurs pendant les mauvaises saisons. Cependant, aucun de ces programmes n'a cours à Las Coloradas et ses habitants ne peuvent bénéficier de ces sources de revenus supplémentaires. Il se pourrait que, la pêche n'étant pas reconnue comme l'activité principale à Las Coloradas, les pêcheurs de cette communauté perdent en visibilité et, du coup, leur éligibilité.

Malgré tout, des groupes intéressés par des projets de conservation environnementale ont été créés à Las Coloradas. Ainsi, un habitant de Las Coloradas ainsi que des femmes regroupées en association ont pu participer à des projets de développement en lien avec l'environnement par l'entremise d'un représentant du Programme des Nations Unies pour le développement (PNUD). Le premier individu a été mis en contact avec l'Agence de coopération internationale du Japon (JICA), laquelle lui a offert des gaminets (tee-shirts) à l'effigie de l'organisme, du matériel scolaire et des programmes éducatifs conçus pour instruire les enfants au sujet du développement durable. Le projet a aussi permis la construction de toilettes publiques dont les murs extérieurs ont été peints d'images naïves évoquant la flore et la faune locales, mais d'autres initiatives ne s'en sont pas suivies.

Le projet proposé aux femmes, et soutenu financièrement par les Nations Unies, visait quant à lui la production de légumes biologiques. La dizaine de femmes aurait cultivé un grand potager commun avec l'objectif de produire localement des aliments sains. Elles se lancèrent dans l'aventure avec enthousiasme. Elles furent cependant rapidement confrontées aux problèmes de propriété foncière : l'accès à la terre leur fut refusé, bien qu'elles firent la demande à la salinera et au municipe. En dernier recours, elles durent 
donc chacune, individuellement, faire leur jardin sur leur propre terrain. L'une d'elle explique :

«Évidemment, la Réserve ne peut te donner un terrain, les terrains de la Réserve sont nationaux, ils sont du pays. De plus, il n'y a plus d'ejidos ${ }^{5}$ par ici. »

\section{Les ressources ligneuses, vision sylvicole et régulations} particulièrement litigieuse à Las Coloradas. D'abord, la compréhension des lois en vigueur est très différente selon les interviewés. Certains prétendent que des permis «d'extraction » existent, et qu'il suffit de les demander. Ces derniers ne voient pas non plus d'entrave morale à se servir dans les boisés aux environs du village. D'autres, tout de même, considèrent qu'il y a une distinction entre la récolte du bois sec et celle du bois vert. Enfin, d'aucuns affirment que toute coupe est interdite, et que cet état de fait frappe négativement d'abord les plus démunis : «Il y en a plusieurs ici qui sont pauvres, ce dont ils ont besoin, c'est du bois parce qu'ils n'ont pas de four. »

Une certaine vision sylvicole semble intrinsèque à une esthétique de la forêt, comme l'explique un habitant:

«Prenons vous-même par exemple, n'allez-vous pas voir le coiffeur, ne devez-vous pas vous couper les cheveux ? Dans ce cas, c'est pareil avec les plantes, il faut lui couper les cheveux pour qu'elle grandisse de plus belle. Les gens de la Réserve me répondent: «Ce n'est pas ce qu'il faut faire avec la nature »; parfois ils se mettent en colère parce qu'en plus ils ont étudié, mais ils n'ont jamais analysé les choses qui peuvent nous manquer, parce que nous en avons besoin pour construire. Ce n'est pas pour vendre. Nous faisons de ces plantes un usage adéquat et elles poussent toujours bien belles ».

La collecte spontanée du bois aurait aussi, selon cette vision sylvicole, la vertu de limiter les risques d'incendies de forêt. Les habitants de Las Coloradas remarquent d'ailleurs les changements dans la structure de leurs forêts que la conservation environnementale stimule : ils s'inquiètent de l'augmentation de la densité de biomasse combustible dans les boisés qu'ils avaient coutume de nettoyer.

Les habitants s'indignent aussi du fait que les sanctions contre les coupes soient variables et que l'application des lois soit relâchée en raison du manque de ressources financières et humaines dont dispose le procureur fédéral de la protection de l'environnement pour remplir son mandat.

D'autre part, des habitants de Las Coloradas attestent que des braconniers de bois viennent sur leur territoire y couper du bois illégalement ainsi que pour voler des plants de palmiers (kuka, chiit), des essences recherchées dans les centres touristiques de même que pour les maisons secondaires de villégiateurs fortunés. Les habitants se plaignent cependant que ces spoliations demeurent généralement impunies. Ses moyens étant en outre limités, le procureur invite la population à le contacter pour témoigner des infractions. Cependant, les habitants jugent que cette responsabilité peut avoir des conséquences dangereuses et ne devrait pas leur échoir.

\section{Le sel : écologie et discours environnementalistes}

Comparé à l'impact environnemental des aménagements de la salinera, la coupe de bois pour usage «traditionnel » ou domestique paraît aux membres du village très peu 
dommageable. Les habitants font observer que le long des charcas de sel, les palétuviers se dessèchent et meurent ; en agrandissant les salines, l'entreprise a un impact radicalement plus destructeur que leurs coupes partielles. Les habitants de Las Coloradas, en particulier ceux qui ont travaillé dans des projets environnementaux ou pour la Réserve, ne peuvent se résoudre à accepter le clivage entre ce qui est permis à la salinera et les contraintes que leur sont imposées.

Selon les habitants, l'appropriation de terrains par la compagnie salinière se fait avec l'appui de la Réserve, suscitant plusieurs incompréhensions et insatisfactions. Il est à noter que la carte de la Réserve ne rend pas compte des limites de la concession d'ISYSA et n'établit pas de zone tampon entre cette concession et le village, d'où les résidents de ce dernier pourrait tirer du bois. Voici les propos d'un homme puis d'une femme du village :

«L'entreprise avance, avance, avance, mais ça se fait avec l'accord du SEMARNAT, avec sa supervision. Nous, pour couper une branche de huano pour faire notre maison, nous avons beaucoup de problèmes et nous voyons que l'industrie fait ce qui lui plait. Regardez leur technique pour avancer, ils mettent les résidus du bassin de sel autour, la végétation est tuée, évidement, elle sèche, elle est morte parce qu'ils l'ont tué. Ensuite, rapidement, ils l'arrachent avec les machines et le sel monte.

L'industrie du sel, ils détruisent, mais pour eux c'est comme si de rien n'était. Mais en fait, c'est terrible de voir que même quand tu rapportes la destruction de plantes à la Réserve, ils le font à la tombée du jour, ils détruisent les plantes et ils font de nouveaux bassins, la Réserve ne te donne même pas de réponse, comme si de rien n'était ».

Malgré l'éradication de la mangrove dans son emprise, l'entreprise salinière affiche un discours écologique et collabore à de nombreuses initiatives de la Réserve, du SEMARNAT et de différentes ONG. Sur son site web, elle indique, d'une part, sa collaboration avec la société Audubon depuis les premières études sur les populations de flamants roses en 1950 (ISYSA n.d.). D'autre part, l'entreprise dit aussi produire 35000 plantes par année afin de permettre la reforestation des dunes côtières. L'entreprise s'approprie aussi la paternité du programme de protection des tortues. Enfin, elle se targue d'être une entreprise qui a la protection de l'environnement à cœur et affirme que la « Certification industrie propre » qui leur a été décernée par un programme national en est la preuve. Ce discours environnementaliste est présent dans l'ensemble des communications émanant de la compagnie. Dans la Conférence inaugurale sur l'importance des salines par évaporation solaire, un employé de la réserve témoignait que :

«ISYSA collabore aux principaux projets de conservation de la Réserve de Biosphère de Ría Lagartos notamment afin d'approfondir et de diffuser la connaissance au sujet de la conservation des ressources naturelles, compte tenu que les ressources qui sont importantes pour l'économie de la région le sont aussi pour les salines » (Ortiz-Milan 2006, traduction libre).

L'entreprise ne manque pas, par ailleurs, de défendre ses intérêts lorsqu'elle est impliquée dans un potentiel litige autour de l'utilisation de l'environnement. Ainsi, le document issu des consultations publiques qui eurent lieu en 2006-2007 (en lien avec la révision du Plan de gestion de la Réserve), nous informe des manœuvres d'ISYSA pour modifier le texte du Programme de conservation et de gestion de la CONANP afin de préserver son image. En particulier, l'entreprise a demandé à ce que soit retirée du programme la phrase «Sans aucun doute, au cours de son histoire l'industrie saline [au 
même titre que la croissance urbaine] a été source d'impacts environnementaux qui présentent encore aujourd'hui des effets et des risques. » (CONANP 2007).

Bien que l'entreprise entretient de bons liens avec la Réserve de biosphère, cette dernière demeure vigilante quant aux ambitions de la salinière d'agrandir l'emprise de ses bassins. Smardon rapporte qu'au cours des années 1990, les gestionnaires de la Réserve eurent vent du projet de l'usine de s'étendre tout en doublant sa production pour atteindre le million de tonnes par an. La volonté du Secrétariat de l'écologie et du développement urbain de stopper toute expansion non autorisée de la compagnie a suscité de la résistance de la part des propriétaires de l'usine. À l'été de 1990, le Secrétariat coupait l'alimentation des bassins d'évaporation, entrainnant du coup la coupure de l'approvisionnement en eau potable au village, qui provient de l'usine. Il n'en fallait pas plus pour que la communauté pense que le Secrétariat les menaçait directement, faisant par le fait même resurgir des tensions latentes entre le syndicat des travailleurs du sel et les associations de pêcheur (Smardon 2009).

\section{"Nos flamants roses, notre plage »: les entraves administratives et spatiales à l'essor du tourisme}

50 Le tourisme est une activité économique peu développée à Las Coloradas. Cette situation n'est pas causée par un manque d'intérêt de la part de la population, au contraire, mais plutôt par une quasi-impossibilité de développer cette activité en raison, d'une part, des contraintes d'accès à la propriété des terres pour y installer les infrastructures nécessaires et, d'autre part, par le « détournement » et « l'accaparement » de ces activités et des touristes dans le village de Río Lagartos, selon les habitants de Las Coloradas.

51 Les habitants de Las Coloradas, et tout particulièrement ceux dont la maisonnée ne compte pas d'employé dans l'usine de sel, souhaitent vivement que des activités touristiques prennent place dans le village. Ils affirment que c'est la seule alternative à la production de sel puisqu'ils n'ont pas d'ejidos permettant la pratique de l'agriculture ou l'élevage et que la pêche, en déclin, leur est limitée à la capture des espèces les moins lucratives.

52 Les habitants prétendent que c'est dans leur village que sont situées les meilleures plages de la région. En effet, Río Lagartos et San Felipe, bien qu'étant des villages côtiers, ne sont pas édifiés directement sur une plage : les touristes ne peuvent pas se baigner dans la mer qui leur fait face. À Las Coloradas, au contraire, le village s'étend le long d'une belle et longue plage au sable fin.

Les habitants disent aussi que les flamands roses, emblème de la Réserve de biosphère et principale raison de la visite des touristes, se trouvent majoritairement exactement dans leur village, car les oiseaux viennent s'y nourrir des artémias qui peuplent les bassins d'évaporation du village. Ils revendiquent ces oiseaux comme étant les leurs et déplorent que ce ne soit pas eux qui bénéficient de la manne de touristes qui viennent les observer. Ils critiquent les promoteurs d'écotourisme de Río Lagartos qui amènent les visiteurs en embarcations jusque dans la zone de la lagune adjacente aux bassins de sel de leur village pour y guider des excursions vouées à l'observation des flamands.

Pour sa part, la famille Roche refuse de céder une partie de sa concession à des fins de développement touristique qui pourrait inclure quelques restaurants, des hôtels modestes et des palapas ${ }^{6}$. La Réserve de biosphère n'a pas quant à elle d'autorité légale 
pour octroyer des terrains et le municipe de Río Lagartos refuse d'accorder des permis de construction sur son fondo legal, un espace dédié à l'habitation et aux infrastructures essentielles du village. Certains habitants racontent que le municipe maintiendrait cette position car ses administrateurs ne voudraient pas que Río Lagartos perde une partie de sa clientèle au profit de Las Coloradas.

Une femme de Las Coloradas a malgré tout pris l'initiative de construire des petites maisonnettes en bois et en feuilles de palmier sur son propre terrain afin d'héberger les touristes, préférablement étrangers. Toutefois, aucun client n'a été observé pendant nos séjours qui se déroulaient de mai à août. Au moins une autre femme souhaite elle aussi accueillir des touristes sur des terres du village :

«Je vous le dis, si nous demandons un permis et qu'ils ne nous le donnent pas, nous allons le faire quand même et nous gérerons les problèmes en temps venu, mais nous le ferons, même sans permis ".

Toutefois, à ce jour, ce désir ne s'est pas réalisé.

Figure 5 : Plage de sable blanc du village de Las Coloradas où les restes de la structure de l'ancien quai sont toujours saillants

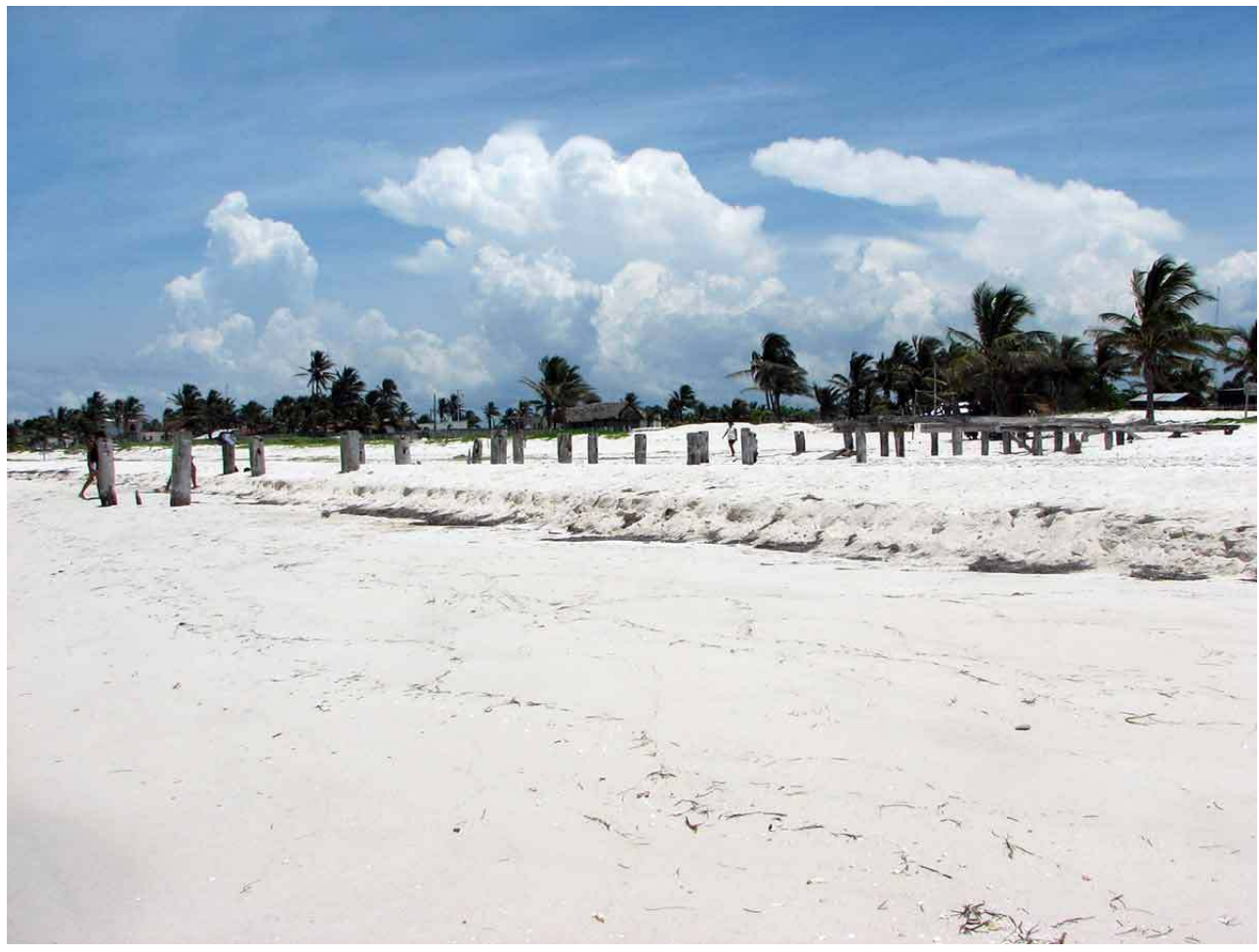

(c) S. Doyon

\section{Las Coloradas : dynamiques sociales et espaces à s'approprier et à protéger}

Les habitants de Las Coloradas perçoivent leur espace comme étant restreint telle une oasis, petite mais porteuse de projets d'avenir, et ils estiment avoir des droits sur celui-ci. Toutefois, cet espace se raréfie et se politise; les incertitudes quant aux droits et aux titres 
de propriété de chacun et la présence de la Réserve ont notamment contribué à ce phénomène.

D'abord, l'espace marin, marqué par la pêche, est saturé de conflits. Le lieu de la pêche est un enjeu, l'étendue face au village de Las Coloradas étant réclamée pour l'usage exclusif de ses propres pêcheurs. Les espèces le sont aussi, les pêcheurs réclamant les mêmes privilèges que les autres pêcheurs actifs sur la côte, particulièrement pour la lucrative pêche à la langouste. Bien qu'ils aient joint les rangs des pêcheurs plus tard que les autres en raison de leur statut initial défini et délimité par le rancho ainsi qu'en raison de leur prospérité relative issue du travail dans l'industrie du sel, les gens de Las Coloradas estiment qu'ils méritent le droit de pêcher toutes les espèces présentes chez eux, comme les autres.

L'espace terrien est aussi réclamé par les habitants de Las Coloradas. Coincés entre les propriétés de l'usine de sel et l'espace administratif du municipe de Río Lagartos, les habitants disent sentir le besoin d'avoir des petites parcelles de terre afin d'y pratiquer l'agriculture ou l'élevage. N'en disposant pas, les gens pratiquent un maraîchage modeste sur leurs propres terrains et laissent leurs animaux paître librement dans le village. Puisque les chèvres et cochons causent parfois des dégâts, cette situation génère elle aussi des conflits. D'après un employé municipal de 45 ans,

«La Réserve empêche d'avoir des terres pour l'élevage ; nous devons [en attendant] vivre dans les odeurs de nos animaux. Les habitants souhaiteraient aussi avoir l'opportunité de pouvoir faire l'élevage de bovins, comme c'est le cas ailleurs dans la région ».

60 Les résidents de Las Coloradas ressentent aujourd'hui les conséquences de leur consentement à habiter sur une concession pendant des décennies, les réformes agraires enclenchées après la révolution n'ayant pas attribué d'ejido au rancho de Las Coloradas. Palliant ce manque perçu, certains habitants, notamment de jeunes retraités de l'usine, ont acquis avec leurs primes de petits terrains pour l'agriculture dans l'ejido de Loche, à 43 kilomètres de Las Coloradas.

61 Alors que les habitants sentent que la compagnie salinière avance impunément sur le territoire, ils disent ne pouvoir disposer de plus de terre pour faire un jardin ou un projet touristique à petite échelle. Les règles sur l'utilisation du bois sont elles aussi perçues comme des contraintes injustes et disproportionnées, car ils considèrent que ces règles ne sont pas appliquées à la compagnie salinière, la Réserve lui laissant le champ libre et la vigilance faisant défaut.

62 La problématique de l'occupation de l'espace est aussi difficile pour les Coloradeños en raison des rapports avec Río Lagartos, le chef-lieu du municipe, particulièrement en ce qui concerne la répartition géographique du développement du tourisme et les contraintes d'utilisation des terrains municipaux. À cela s'ajoute les problèmes d'approvisionnement en eau qui sont chroniques dans le municipe. Tous ces irritants sont, selon les Coloradeños, exacerbés par les allégeances politiques différentes entre ces deux localités. Lors des élections de 2006, de la violence et de l'intimidation à l'endroit des représentants du parti politique adverse à celui du chef-lieu ont été perpétrées, notamment des coups de feu à proximité des résidences de leurs supporteurs de Las Coloradas.

63 Toutes ces contraintes sont sources d'insatisfactions pour les habitants de Las Coloradas qui souhaitent investir cet espace de leurs projets : 
« Tout ce qui est près de la Ría est en concession, il y a la Réserve, la langouste dans la mer est aussi sous-concession, c'est une activité sous-concession parce que tu ne peux pas la capturer. Nous ne pouvons pas non plus prendre un petit morceau de terrain parce que cela appartient à l'entreprise. Donc, nous ne pouvons rien faire ».

\section{Espace et protection de l'environnement} dans une aspiration à développer le tourisme. Une dizaine de personnes dans le village, qui ont la caractéristique de ne pas travailler à l'usine de sel, s'attachent à défendre activement les intérêts et objectifs de la Réserve; ils sont même en faveur d'un durcissement des règles de protection de l'environnement afin que cela puisse favoriser l'émergence d'un écotourisme solide, profitable et durablement structurant pour le village. Toutefois, ils notent qu'il n'y a pas suffisamment de transmission de connaissances et qu'il n'y a pas suffisamment de moyens de surveillance déployés. Deux hommes racontent :

«Que dois-je faire ? Surveiller ? Et puis je le léguerai à mes fils, à mes petits-fils, qu'ils puissent aller là (dans la Réserve) où il $\mathrm{y} a$ du gibier, des jaguars... et bien cela ne se pourra pas, parce qu'ils n'emprisonnent personne, ils n'envoient pas de surveillants.

Bien... je pense qu'il manque, premièrement, de surveillance, que les lois soient plus rigides et qu'il y ait plus d'informations. Que l'on fasse plus la promotion de l'environnement, comme dans des groupes écologistes, parce que ça n'a l'air de rien, mais quand nous avons travaillé avec les enfants, ils ont gardé un peu de conscience environnementale et on voyait le changement, parce qu'ils en parlaient à leurs parents. Mais il ne faut pas que ce soit seulement une période, il faut du suivi de la part du SEMARNAT et de la part de toute institution, non ? Et pas seulement aux enfants, aux adultes aussi, aux pêcheurs et à tous. Et comme je disais, les lois doivent être plus rigides parce que c'est facile de les enfreindre ».

Les préoccupations environnementales des Coloradeños sont donc constamment animées de désirs et d'aspirations où la qualité de l'avenir de leur village est en jeu, un avenir qu'ils comprennent dépendre à la fois des pratiques productives, des arrangements territoriaux et des relations régionales. 
68 dans l'accès à l'espace : le pôle lié aux activités de pêche, marin, un pôle lié aux activités $\mathrm{du}$ sel, terrien, et un autre lié aux activités de conservation qui chevauche à la fois les espaces marin et terrien, et qui intègre définitivement le littoral. Ces pôles correspondent à la fois à des pratiques bien concrètes, à des représentations de la nature, à des politiques gouvernementales et les investissements et les programmes qui leur sont tributaires. Ils correspondent à des « régimes de nature » qui se croisent désormais, une approche de la nature extractiviste et productiviste, propre au système capitaliste, intègre désormais un régime de nature qui place la conservation de la nature en son centre grâce à des lois, des politiques et des ressources mis à sa disposition et misant sur la valeur des paysages et de la conservation, comme divers auteurs l'ont rapporté dans d'autres contextes (Sabinot \& Doyon 2014, Brockington \& Duffy 2010, Escobar 1999). Il semble cependant que ce régime de nature tend à marginaliser les habitants du village, et tout particulièrement les pêcheurs.

\section{Conclusion}

69 À Las Coloradas, les relations socio-environnementales sont marquées par l'espace côtier qu'a d'abord défini l'usine de sel. La vie des habitants est rythmée par les quarts de travail, les lieux d'occupation et d'utilisation de la nature dans les salines, le patrimoine foncier et la configuration même du village, héritée d'une période fondatrice où ce lieu était encore un rancho sans structure urbanistique. Leur quotidien et leur avenir s'accrochent également à des revendications d'une valorisation de la nature qui leur permettrait d'assurer leur survie dans un contexte où les pressions se font toujours plus importantes.

70 L'usage et l'accès aux ressources est clairement au cœur des relations entre les habitants $\mathrm{du}$ village et des institutions y étant implantées. L'analyse de cet usage en souligne les dimensions historiques et scalaires, de même que les politiques étatiques et nationales influençant les représentations qu'ont chacun des acteurs de cette dynamique. Ainsi, on note que le « régime de nature » dominant la côte et le village de Las Coloradas s'est logé pendant des décennies à l'intérieur d'un cadre capitaliste faisant la promotion de l'exploitation et de l'extraction des ressources naturelles. De plus en plus cependant, les principes qui guident la conservation environnementale s'inscrivent dans les préoccupations des activités de pêche et de production salicole, les uns insistant sur le caractère écologique de leur pêche, les autres sur les «qualités ancestrales » d'une production locale s'inspirant des antiguos Mayas et sur les qualités de bon citoyen corporatiste faisant la promotion de la conservation environnementale et contribuant aux activités de la réserve de biosphère. Ces articulations entre les activités d'exploitation et de conservation semblent être en partie ancrées dans une motivation à maintenir un accès aux ressources ou à en revendiquer un, instrumentalisant en partie les questions de conservation pour d'autres intérêts, mais fédérant les discours, les pratiques et les politiques autour de la conservation environnementale. Cette dynamique s'ancre dans l'émergence d'un régime de conservation de la nature caractérisé par une logique « où les institutions sociales, économiques et politiques confluent dans la conservation de l'environnement en mobilisant des ressources (économiques, politiques, administratives, humaines), des technologies, des lois, des politiques, des discours et des pratiques qui forment un assemblage de plus en plus cohérent au service des valeurs et des intérêts

Revue d'ethnoécologie, 14 | 2018 
environnementaux» (Doyon 2013). Cette mécanique contribue à la marginalisation croissante des activités de pêche dans l'économie nationale et régionale du Yucatán et à la marginalisation économique, écologique et sociale des pêcheurs et de leur famille, tout particulièrement à Las Coloradas où, pour des raisons historiques, les organisations de pêcheurs ont moins de pouvoir en raison, notamment, de leur interdiction d'accès aux espèces les plus lucratives. Ainsi, un espace marqué par la prégnance de rapports socioenvironnementaux articulés aux principes de la conservation qui s'incarne dans une réserve de biosphère semble maintenir des rapports sociaux iniques, renforçant certaines des hiérarchies socio-économiques déjà instaurées depuis plusieurs décennies. La production de paysages et d'une nature destinées à l'écotourisme transformera-t-elle cette dynamique et offrira-t-elle une oasis aux pêcheurs?

\section{BIBLIOGRAPHIE}

Breunig L.A. 2006 - Conservation in Context: Establishing Natural Protected Areas during Mexico's Neoliberal Reformation. Thèse de doctorat, Tucson, Department of Geography and Regional Development, University of Arizona.

Brockington D. \& Duffy R. 2010 - Capitalism and conservation: the production and reproduction of biodiversity conservation. Antipode 42 : 469-484.

CONANP 2007 - Processo de consulta publica del programa de conservación y manejo de la Reserva de la Biosfera Ría Lagartos. [En ligne] URL : http://www.conanp.gob.mx/anp/consulta/cuadro\% 20consulta.doc Consulté le 16 octobre 2010.

CONANP 2010 - ¿Qué hacemos? Áreas protegidas decretadas. [En ligne] URL : http:// www.conanp.gob.mx/que_hacemos/Consulté le 16 octobre 2010.

Doyon S. 2013 - Une révolution de l'environnement : ethnographie d'un village côtier à Cuba. Québec, Presses de l'Université Laval.

Doyon S., Guindon A. \& Leblanc C. 2008 - Descentralización, regionalización y atomización en Río Lagartos, Las Coloradas y El Cuyo : Prácticas y políticas de las comunidades del oriente de Yucatán. In : Fraga J., Villalobos G., Doyon S. \& García A. (Ed.), Gobernanza Costera en México : Descentralización y Manejo Ambiental en la Península de Yucatán. Plaza y Valdés, Universidad Autónoma de Campeche : 239-257.

Doyon S. \& Paquet P.-A 2015 - Las Coloradas : salines, enclavement foncier et aspirations territoriales. In : Doyon S. \& Sabinot C. (Ed.), Anthropologie des espaces côtiers et de la protection environnementale: Pêche, sel et flamants roses dans les aires protégées yuquatèques au Mexique. Québec, Presses de l'Université Laval : 197-222.

Escobar A. 1999 - After Nature: Steps to an Antiessentialist Political Ecology. Current Anthropology 40 (1) : 1-30.

Fraga J. 1999 - Política ambiental y relaciones de género en una área natural protegida: la relación global/ local en Río Lagartos, México. Thèse de doctorat, Québec, Département d'anthropologie, Université Laval. 
Fraga J. 2004 - Los habitantes de la zona costera de Yucatán : entre la tradición y la modernidad. In : Arriaga E.R., Villalobos Zapata G.J., Azuz Adeath I. \& May F.R. (Ed.), El Manejo Costero en México. Campeche, Universidad Autónoma de Campeche, SEMARNAT, CETYS-Universidad, Universidad de Quintana Roo : 497-506.

Fraga J., Salas S. \& Mexicano-Cíntora G. 2008 - La pesca en Yucatán : de la abundancia a la escasez, a la fragilidad de las estructuras institucionales. In : Fraga J., Villalobos G., Doyon S. \& García A. (Ed.), Gobernanza Costera en México : Descentralización y Manejo Ambiental en la Península de Yucatán. Plaza y Valdés, Universidad Autónoma de Campeche : 179-201.

INEGI 2010 - Conteo de población y vivienda. Yucatán. Gobierno de Mexico.

ISYSA n.d. - Ecologia, [En ligne] URL : http://www.isysa.com.mx/flash/indexcosysa.htm Consulté le 16 octobre 2010.

Lapointe M. 2006 - Histoire du Yucatán XIX ${ }^{e}-X X I^{e}$ siècle. Paris, L'Harmattan.

Mansfield B. 2001 - "Modern" industrial fisheries and the crisis of overfishing. In : Peet R., Robbins P. \& Watts M.J. (Ed.), Global Political Ecology. New York, Routledge : 84-99.

Ortiz-Milan S.M. 2006 - Contribution of industrial salinera de Yucatán (ISYSA) to the protection of the environment in the reserve of the biosphere Rio Lagartos, Yucatán, Mexico. First International Conference on the Ecological Importance of Solar Saltworks. Santorini Island, Greece, 20-22 October $2006: 47$.

Peet R., Robbins P. \& Watts M.J. 2011 - Global Political Ecology. London, New York, Routledge.

Sabinot C. \& Doyon S. 2014 - Rapports socio-environnementaux, construction de l'espace, et régimes de nature dans la Réserve de biosphère de Celestún, Yucatán, Mexique. Canadian Journal of Latin American and Caribbean Studies 39 (2) : 262-281.

Smardon R.C. 2009 - Sustaining the World's Wetlands: Setting Policy and Resolving Conflicts. New York, Springer-Verlag.

UNESCO 1996 - Réserves de biosphères : La Stratégie de Séville et le Cadre Statutaire du Réseau mondial. Paris, UNESCO.

Wolf E. 1972 - Ownership and political ecology. Anthropological Quarterly 45 (3) : 201-205, cité dans Biersack A. \& Greenberg J. 2006 - Reimagining Political Ecology. Durham \& London, Duke University Press.

\section{NOTES}

1. Un rancho est une petite agglomération peu peuplée située sur une parcelle privée, des terres d'hacienda ou du domaine public (Lapointe,2006:107).

2. La pêche offre depuis une alternative aux travailleurs du sel et aux habitants de Las Coloradas en général. Il n'est pas rare que les employés licenciés par l'usine pour retards fréquents ou insubordination, de même que ceux qui quittent car ils ne supportent pas le rythme, l'horaire et la discipline du travail dans les salinières, se tournent vers la pêche.

3. Le commerce d'œufs de tortues marines est illégal, néanmoins, il existe un marché pour ce bien et Las Coloradas est reconnu comme un lieu où il est possible de s'en procurer.

4. Bien que l'entreprise ISYSA soit devenue une société anonyme (S.A de C.V) ne relevant plus directement de la famille Roche (bien que plusieurs de ses membres soient encore des dirigeants de l'entreprise), les habitants du village continuent de dire que c'est l'entreprise des Roche. 
5. Un ejido est une propriété foncière tenue collectivement par les paysans d'un village. L'usufruit des terres ejidales interdisait leur vente ou leur cession, constituant un des principaux gains de la révolution mexicaine. Toutefois, depuis les modifications constitutionnelles de 1992, leur privatisation et leur division a été légalisée.

6. Petit abri, constitué d'un toit de bois et de feuilles de palmier; ils sont très populaires comme structure d'accueil pour les touristes.

\section{RÉSUMÉS}

$\mathrm{Au}$ Mexique, les intérêts et les activités qui se déploient dans les régions littorales se multiplient. À la pêche côtière traditionnelle s'ajoutent notamment le tourisme de masse, l'écotourisme ainsi que des activités de conservation environnementale. Cette situation n'est pas étrangère aux côtes du Yucatán et cet article propose d'en examiner les enjeux. Par le truchement d'une perspective critique et diachronique, cet article examinera les rapports socio-environnementaux à l'œuvre au sein d'un village logé dans la réserve de biosphère de Ría Lagartos, se situant à la croisée de processus qui tendent à marginaliser socialement et économiquement ses habitants, et plus particulièrement les pêcheurs côtiers, les enclavant au sein de l'aire protégée. Basé sur un travail ethnographique qui se fonde dans l'approche de la political ecology, cet article analyse l'occupation et l'utilisation du littoral à Las Coloradas et met en lumière le rôle imparti aux différentes activités économiques qui s'y pratiquent - la pêche, la production salicole, le tourisme et la conservation environnementale - au sein des processus de contestation et de négociation des espaces marin, littoral et terrien qui s'y construisent.

In Mexico, the variety of activities and concerns that are currently being deployed along the coasts is growing. In addition to the traditional fisheries coastal fisheries, mass tourism, ecotourism and environmental conservation are now part of the landscape and actively reshaping it. As similar developments also impact the coasts of Yucatán, this article examines the stakes, given the recent transformations affecting this region. Through a critical and diachronic perspective, this article explores the socio-environmental relationships at work in a village located within the Ría Lagartos Biosphere Reserve as well as the processes that are socially and economically marginalizing its inhabitants, and more particularly the local fishermen whose livelihoods are closely tied to the coastal areas now enclaved within the protected area. Based on ethnographic research and a political ecology framework, this article analyzes sea and land uses along the coasts of Las Coloradas. This article also highlights the role of various economic activities - including fisheries, the salt industry, tourism, and environmental conservation - in shaping ongoing negotiations and disputes about coastal and land uses in this region.

\section{INDEX}

Keywords : anthropology of conservation, protected areas, fisheries, coasts, political ecology, Mexico, Yucatán

Index géographique : Yucatán, Mexique

Mots-clés : anthropologie de la conservation, aires protégées, pêche, côtes, écologie politique, Mexique, Yucatán 


\section{AUTEURS}

\section{SABRINA DOYON}

Université Laval - Québec, Canada

\section{PIERRE-ALEXANDRE PAQUET}

University of Wisconsin-Madison - Québec, Canada 